\title{
Clinical course and cardiovascular outcomes in patients with the long-chain 3-hydroxyacyl- -coenzyme A dehydrogenase deficiency
}

\author{
Joanna Kwiatkowska ${ }^{1}$, Jolanta Wierzba ${ }^{2}$, Anna Karaszewska ${ }^{2}$, \\ Dariusz Kozlowski ${ }^{3}$, Jolanta Sykut-Cegielska ${ }^{4}$, Agnieszka Stanko ${ }^{1}$ \\ ${ }^{1}$ Department of Pediatric Cardiology and Congenital Heart Defect, Medical University of Gdansk, Poland \\ ${ }^{2}$ Department of General Nursery Department of Pediatrics, Hematology, Oncology, \\ Medical University of Gdansk, Poland \\ ${ }^{3}$ Department of Cardiology, Medical University of Gdansk, Poland \\ ${ }^{4}$ Screening and Metabolic Diagnostics Department, The Institute of Mother and Child, Warsaw, Poland
}

\begin{abstract}
Long-chain 3-hydroxyacyl-coenzyme A dehydrogenase deficiency (LCHADD) is a rare inborn metabolic disease with the significant cardiac involvement, but remains without an established frequency of its occurrence. Its pathophysiology has not been fully understood or explained until now [1-3]. In the literature, there are sparse reports about cardiac symptoms in LCHADD [4-7]. Inborn errors of metabolism could often present with cardiomyopathy as it does with other diseases such as mitochondrial and lysosomal storage $[8,9]$.

Seventeen children with LCHADD confirmed genetically at the average age of 6 months (range, 0.1-13.0 years) were admitted and were under regular follow-up. They were all from 15 unrelated families in the Kashubian area of Poland (Table 1). Molecular analysis of gene coding for mitochondrial trifunctional protein revealed $1528 \mathrm{G}>\mathrm{C}$ mutation of the HADHA gene (locus $2 \mathrm{p} 23$ ) in all of them. Two cases (patients 10,11) were detected through newborn screening by tandem mass spectrometry. In the cases of two other children (patients 7,8 ), the suspicion of LCHADD was established because of the positive family history. The other patients were diagnosed after the first episode of clinical decompensation. Cardiac abnormalities were detected in 15 of $17(88.2 \%)$ patients (Table 1$)$. Cardiomyopathy was diagnosed in $11(64.7 \%)$ children. In 2 cases (patients 9,16 ), both types of cardiomyopathies, dilated cardiomyopathy (DCM)
\end{abstract}

followed by hypertrophic cardiomyopathy, were disclosed in the follow-up period [5]. One initially healthy child (patient 11) with no echocardiographic evidence of any type of cardiomyopathy, developed an acute DCM and died a few hours later after the first symptoms of decompensation; DCM was confirmed by autopsy. Four children (patients 10, $12,14,15)$, with detected DCM at the time of diagnosis of LCHADD, manifested the normalization of echocardiographic image during the follow-up. Seven other patients (patients 2, 4, 6, 7, 8, 11, 17) from this study group had no echocardiographical evidence of any type of cardiomyopathy up to their final follow-up visit at the end of the study. The cardiomyopathies were not the only cardiac anomalies observed in these patients. One child (patient 7) was diagnosed with intermittent ventricular preexcitation on $24 \mathrm{~h}$ Holter-electrocardiography monitoring and one girl (patient 1 ) with prolongation of QTc. In the other 8 children (patients 2, 6, 8, 9, 12, $14,15,16)$ unspecific repolarization abnormalities were identified during the follow-up period. Additionally, in one child (patient 13) coarctation of the aorta was diagnosed. All children maintained a low-fat diet with medium-chain triglycerides (MCTs) supplementation (compliance 95-100\%) and standard heart failure treatment if necessary.

As it can be seen only 4 patients were diagnosed directly after screening or after positive family history (patients $7,8,10,11$ ). The remain-

Address for correspondence: Joanna Kwiatkowska, MD, PhD, Department of Pediatric Cardiology and Congenital Heart Defect, Medical University of Gdansk, ul. Dębinki 7, 80-952 Gdańsk, Poland, tel: +48 5834928 70, fax: +48 583492895 , e-mail: joannak@gumed.edu.pl 


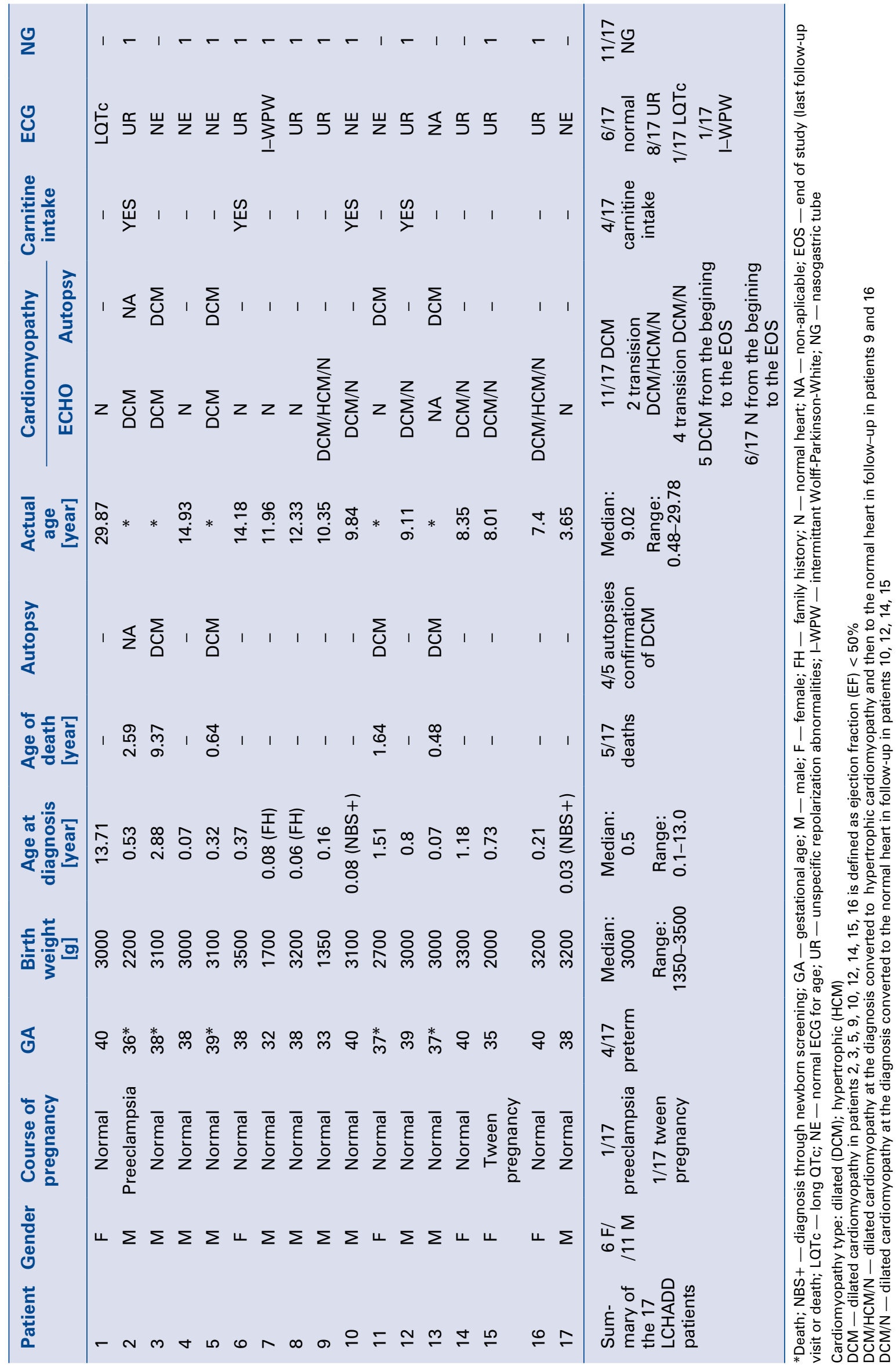


ing patients had the diagnosis established after clinical decompensation. Therefore, only in 4 cases (patients $7,8,10,11$ ) the restriction of fat and MCTs supplementation were included initially with $10-20 \%$ of energy as MCTs and limited long-chain fatty acids intake (10\% of total energy). In the remaining patients, the dietary restriction was included after diagnosis confirmation [2]. In the infantile period nasogastric tube feeding was frequently used but none were fed via percutaneous endoscopic gastrostomy [3]. Only children with documented l-carnitine deficiency received 1-carnitine supplementation (5 out of 17) in doses varying from 5 to $100 \mathrm{mg} / \mathrm{kg} /$ day intermittently.

Five (28\%) patients died during follow-up. There were 3 (patients 2, 5, 13) hospital deaths. The cause of death was the metabolic crisis (triggered by an infection) with a rapid progression of heart failure. It should be emphasized that the other 2 patients (patients 5, 11) demonstrated normal echocardiography before metabolic decompensation, with a sudden development of cardiomyopathy, which was the direct reason for the death at home. In both cases, an autopsy was performed and revealed dilatation of the left heart and histological evidence of DCM.

Living patients age ranged from 3.5 to 29.8 years and follow-up from 3.4 to 16.8 years (median 9.02 years). They remained on treatment and were intermittent symptomatic with an accidental increase of creatinine kinase (CK) levels with no echocardiographic and/or electrocardiographic abnormalities parallel to CK increase. Three of these (patients $4,10,15$ ) demonstrated episodes of rhabdomyolysis parallel to extremely high CK, but without any echocardiographic and/or electrocardiographic abnormalities. We noticed that even with restricting diet most incidents of decompensation proceeded subsequently, even with a short-lived drop in calorie of meals rather than meal composition.

In LCHADD, the metabolism of hydroxy-longchain fatty acids is disturbed. The clinical manifestation depends both on toxicity of hydroxy-long-chain fatty acids esters (which in a cardiac muscle, with myocytes damage, leads to cardiomyopathy and heart arrhythmias) as well as energy production disorder - too small amount of adenosine triphosphate in proportion to energetic needs of cells is produced. The concept assumes that during fasting, the main source of energy production for some tissues, including cardiac muscles, are ketone bodies produced via fatty acid beta-oxidation. This source was not available in LCHADD. The fatal effect of lowering the cellular $\mathrm{pH}[9,10]$ should also be considered.

One of the largest studies of patients with the LCHADD was done by den Boer et al. [11]. That study dated from 2002 , had a cohort of 50 patients and $42 \%$ had cardiomyopathies. In other studies which were conducted in patients diagnosed with LCHADD, the frequency of cardiomyopathies was variable. In another large study of fatty acid oxidation disorders, described 107 patients [12], in $28(26.2 \%)$ cases cardiomyopathies were also reported.

Rarely are the cardiac arrhythmias reported in LCHADD [7], probably because of the sudden death which usually occurs at home with no possibility to monitor the heart rate.

The diagnosis of LCHADD in the presented cohort of children was usually established during the first months of life because of the child's deteriorating health status. Over the last few years, the Tandem-MS screening and the genetic confirmation of the disease have rapidly improved early diagnosis. In general, the recurrent episodes of hypoglycemia, as a consequence of even a short, low caloric intake causes a wide spectrum of cardiac symptoms [5].

Although the cardiomyopathy is not the primary finding in the LCHADD, it can be diagnosed by echocardiography. The electrocardiographic and echocardiographic examinations should be done in every child both with diagnosis of, or with suspicion of LCHADD. At this center, the current cardiological practice is to follow-up these patients regularly at 6-12 month intervals at the outpatient clinic as recommended by Spiekerkoetter et al. [1]. Such a practice can prevent further cardiac complications which is also equally important in the perspective of decompensation process and cardiac death. It is possible that the magnetic resonance images of the heart in these patients when their echocardiography images are normal will play a significant role in our understanding of this phenomenon. Further basic research and multicenter clinical trials are required in order to understand this better.

\section{Conflict of interest: None declared}




\section{References}

1. Spiekerkoetter U, Lindner M, Santer R et al. Management and outcome in 75 individuals with long-chain fatty acid oxidation defects: results from a workshop. J Inherit Metab Dis, 2009; 32: 488-497.

2. Sykut-Cegielska J, Gradowska W, Piekutowska-Abramczuk D et al. Urgent metabolic service improves survival in long-chain 3-hydroxyacyl-CoA dehydrogenase (LCHAD) deficiency detected by symptomatic identification and pilot newborn screening. J Inherit Metab Dis, 2011; 34: 185-195.

3. Piekutowska-Abramczuk D, Olsen RK, Wierzba J et al. A comprehensive HADHA c.1528G $>$ C frequency- study reveals high prevalence of long-chain 3-hydroxyacyl-CoA dehydrogenase deficiency in Poland. J Inherit Metab Dis, 2010; Sep 3 [Epub ahead of print].

4. Martins E, Costa A, Silva E et al. Lethal dilated cardiomyopathy due to long-chain 3-hydroxyacyl CoA dehydrogenase deficiency. J Inherit Metab Dis, 1996; 19: 373-374.

5. Kwiatkowska J, Switonska J, Wierzba J. The recovery from dilated cardiomyopathy followed by hypertrophic cardiomyopathy, in the course of deficiency of long-chain 3 hydroxyacyl-coenzyme A dehydrogebase in infant. JBGC, 2013; 3: 26-28.

6. Dyke PC, Konczal L, Bartholomew D, McBride KL, Hoffman TM. Acute dilated cardiomyopathy in a patient with deficiency of long-chain 3-hydroxyacyl-CoA dehydrogenase. Pediatric Cardiol, 2009; 30: 523-526.

7. Bonnet D, Martin D, Pascale De L et al. Arrhythmias and conduction defects as presenting symptoms of fatty acid oxidation disorders in children. Circulation, 1999; 100: 2248-2253.

8. Tonin AM, Amaral AU, Busanello EN, Grings M, Castilho RF, Wajner M. Long-chain 3-hydroxy fatty acids accumulating in long-chain 3-hydroxyacyl-CoA dehydrogenase and mitochondrial trifunctional protein deficiences uncouple oxidative phosphorylation in heart mitochondria. J Bioenerg Biomembr, 2013; 45: 47-57.

9. Spiekerkoetter U, Wood PA. Mitochondrial fatty acid oxydation disorders: pathophysiological studies in mouse model. J Inherit Metab Dis, 2010; 33: 539-546.

10. Karall D, Brunner-Krainz M, Kogelnig K et al. Clinical outcome, biochemical and therapeutic follow-up in 14 Austrian patients with Long-Chain 3-Hydroxy Acyl CoA Dehydrogenase Deficiency (LCHADD). OJRD, 2015; 10: 21.

11. den Boer ME, Wanders RJ, Morris AA, IJlst L, Heymans HS, Wijburg FA. Long-chain 3-hydroxyacyl-CoA dehydrogenase deficiency: clinical presentation and follow-up of 50 patients. Pediatrics, 2002; 109: 99-104.

12. Saudubray JM, Martin D, de Lonlay P et al. Recognition and management of fatty acid oxidation defects: a series of 107 patients. J Inherit Metab Dis, 1999; 22: 488-502. 